\title{
Baryon Spectroscopy \\ - Recent results from the Crystal Barrel Experiment at ELSA -^
}

\author{
C. Schmidt ${ }^{1, a}$ for the CBELSA/TAPS collaboration \\ Helmholtz-Institut für Strahlen- und Kernphysik, Universität Bonn, 53115 Bonn, Germany
}

\begin{abstract}
To understand the spectrum and the properties of baryon resonances, the CBELSA/TAPS experiment investigates the photoproduction off the nucleon using linearly, circularly polarized or unpolarized photons impinging on a polarized or unpolarized target. Preliminary results on first double polarization observables have been obtained for the reactions $\vec{\gamma} \vec{p} \rightarrow p \pi^{0}$ and $\vec{\gamma} \vec{p} \rightarrow p \eta$ using both circular and linear polarized photons on a longitudinally polarized target. This data will finally provide important information for the partial wave analyzes used to extract the resonances from the data.
\end{abstract}

\section{Introduction}

The non-perturbative regime of QCD - the world of the nucleon and its excitations - is still one of the most exciting challenges in subnuclear physics. Most of the information on the baryon excitation spectrum was obtained from $\pi N$-scattering experiments. These experiments give a clear bias towards resonances with a sizeable $\pi N$-coupling. Recently, our knowledge was supplemented by photo- and electroproduction data offering the opportunity to study resonances with small or vanishing $\pi N$-coupling in reactions like $\gamma p \rightarrow p \eta$. To extract broad and strongly over-
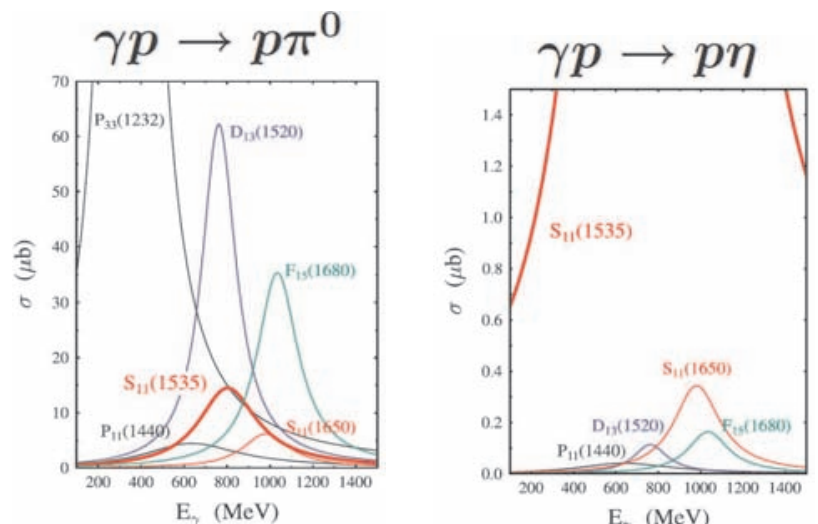

Fig. 1. Sketch of the Breit-Wigner-strength of various resonances in two different finals states. The available phase space is not considered.

lapping resonances from the data two issues are rather important: First, different final states need to be investigated

\footnotetext{
* Supported by the German Science Foundation (DFG) in the frame of the SFB/TR16

a e-mail: schmidt@hiskp.uni-bonn.de
}

since different resonances may couple with rather different strengths to different final states making their observation more probable in certain reactions compared to others (see e.g. Fig. 1). Not only single-meson photoproduction needs to be studied but also photoproduction of the - more complicated - multi-meson final states. The latter ones get more and more important at higher energies as seen in Fig. 2.

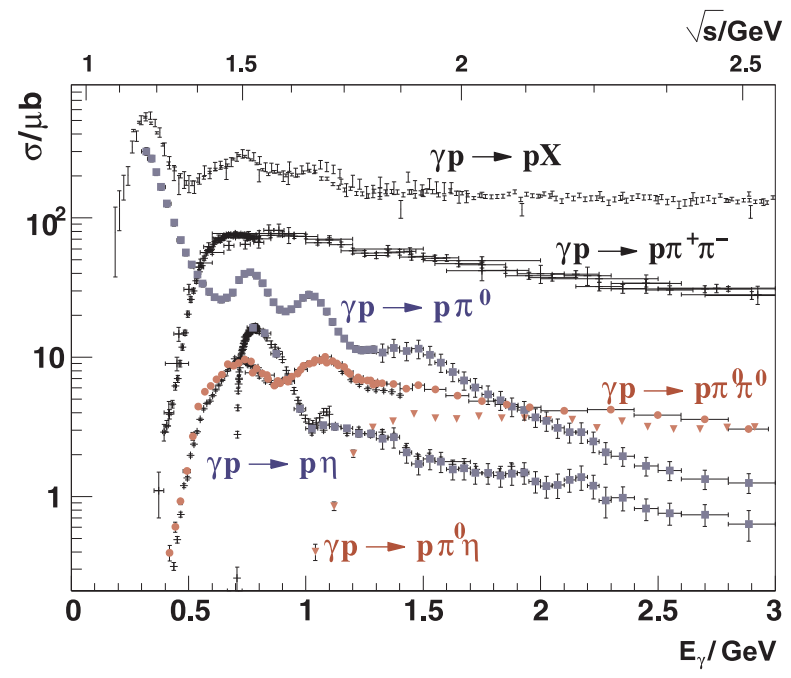

Fig. 2. Total photoabsorption cross section in comparison to various single- and double-meson photoproduction cross sections $\gamma p \rightarrow p X$ [1], $\gamma p \rightarrow p \pi^{+} \pi^{-}$[2,3] (and references therein), $\gamma p \rightarrow p \pi^{0} \pi^{0}$ [4-6], $\gamma p \rightarrow p \pi^{0}$ [7], $\gamma p \rightarrow p \eta[8-10], \gamma p \rightarrow p \pi^{0} \eta$ $[11,12]$

Secondly, polarization observables have to be measured; without polarization observables an unambiguous extraction of the contributing amplitudes from the data will not be possible.

This is an Open Access article distributed under the terms of the Creative Commons Attribution-Noncommercial License 3.0, which permits unrestricted use, distribution, and reproduction in any noncommercial medium, provided the original work is properly cited $\square$ 
EPJ Web of Conferences

\begin{tabular}{|c|c|ccc|ccc|cccc|}
\hline Photon & & \multicolumn{3}{|c|}{ Target } & \multicolumn{3}{c|}{ Recoil } & \multicolumn{4}{c|}{ Target-Recoil } \\
\hline & & & & & $x^{\prime}$ & $y^{\prime}$ & $z^{\prime}$ & $x^{\prime}$ & $x^{\prime}$ & $z^{\prime}$ & $z^{\prime}$ \\
& & $x$ & $y$ & $z$ & & & & $x$ & $z$ & $x$ & $z$ \\
\hline unpolarized & $\boldsymbol{\sigma}$ & 0 & $\boldsymbol{T}$ & 0 & 0 & $\boldsymbol{P}$ & 0 & $T_{x^{\prime}}$ & $-L_{x^{\prime}}$ & $T_{z^{\prime}}$ & $L_{z^{\prime}}$ \\
linear & $(-\boldsymbol{\Sigma})$ & $\boldsymbol{H}$ & $(-\boldsymbol{P})$ & $(-\boldsymbol{G})$ & $O_{x^{\prime}}$ & $(-\boldsymbol{T})$ & $O_{z^{\prime}}$ & $\left(-L_{z^{\prime}}\right)$ & $\left(T_{z^{\prime}}\right)$ & $\left(-L_{x^{\prime}}\right)$ & $\left(-T_{x^{\prime}}\right)$ \\
circularly & 0 & $\boldsymbol{F}$ & 0 & $(-\boldsymbol{E})$ & $\left(-C_{x^{\prime}}\right)$ & 0 & $\left(-C_{z^{\prime}}\right)$ & 0 & 0 & 0 & 0 \\
\hline
\end{tabular}

Table 1. Observables in single pseudoscalar meson photoproduction. Presently accessible are $\sigma, \Sigma, E, G$ (blue) using a longitudinally polarized or unpolarized target and a linear, circular or unpolarized photon beam. $T, P, H, F$ (red): Single and double polarization observables accessible with a polarized or unpolarized beam and transversally polarized target, which is presently in preparation.

\subsection{Polarization observables}

Table 1 shows the 16 observables accessible for photoproduction of single pseudoscalar mesons. For a complete experiment, allowing for a model independent partial wave analysis 8 carefully chosen observables need to be measured [13]. Differential cross sections and the beam asymmetry $\Sigma$ have already been measured for various channels; experiments with a longitudinally polarized target and a circularly or linearly polarized beam have been performed only recently. Experiments with a transversally polarized target are planned for 2010.

The differential cross section for photoproduction of pseudoscalar mesons, using a polarized photon beam and a longitudinally polarized target but without considering the recoil polarization, can be written in the form

$$
\begin{aligned}
\frac{d \sigma}{d \Omega}=\frac{d \sigma_{0}}{d \Omega}(1 & -p_{\text {lin }}^{\text {beam }} \Sigma \cos (2 \phi) \\
& +p_{x}^{\text {target }}\left(-p_{\text {lin }}^{\gamma} H \sin (2 \phi)+p_{\text {circ }}^{\gamma} F\right) \\
& -p_{y}^{\text {target }}\left(-T+p_{\text {lin }}^{\gamma} P \cos (2 \phi)\right) \\
& \left.-p_{z}^{\text {target }}\left(-p_{\text {lin }}^{\gamma} G \sin (2 \phi)+p_{\text {circ }}^{\gamma} E\right)\right)
\end{aligned}
$$

$\sigma_{0}$ represents the polarization independent cross section. The degree of the photon beam polarization is represented by $p_{\text {lin }}^{\text {beam }}$ and $p_{\text {circ }}^{\text {beam }}$. The definition of the angles is illustrated in Fig. 3. The angle between the photon direction $z$ and the outgoing meson defines $\Theta$. For linearly polarized photons, the angle $\phi$ is defined as the angle between the polarization vector of the incoming photon and the reaction plane.

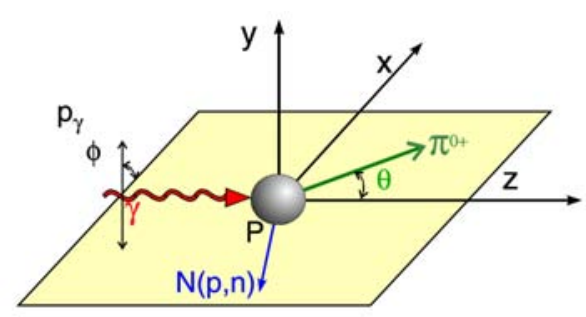

Fig. 3. Single meson production demonstrated on the reaction $\vec{\gamma} \vec{p} \rightarrow p \pi^{0}$.

\section{$2 \eta$-photoproduction}

Photoproduction of $\eta$-mesons is an interesting reaction to investigate the baryon spectrum. The $\eta$ meson as isospin zero particle acts as isospin filter; only baryon resonances with isospin $\mathrm{I}=1 / 2$ can contribute. Close to threshold, in particular the $S_{11}(1535)$ resonance contributes strongly, see Fig. 4. Other resonance contributions have been found to

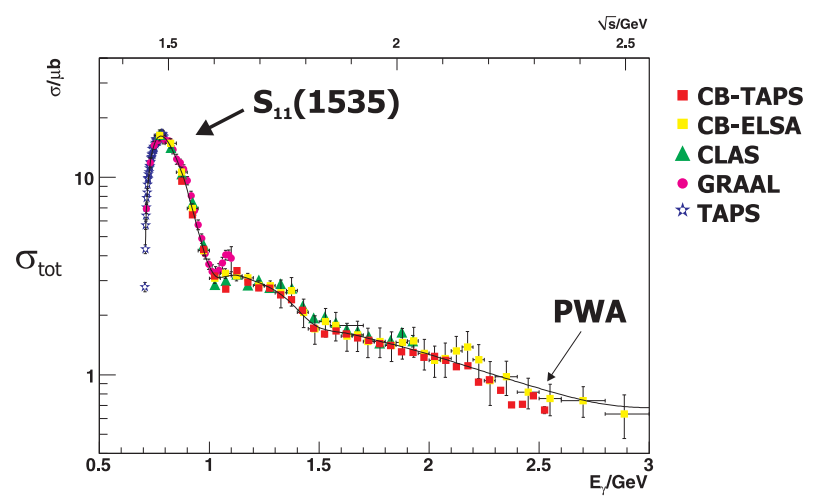

Fig. 4. Total $\gamma p \rightarrow p \eta$ cross section for the CBELSA/TAPSdata [9] in comparison to previous CB-ELSA [14], GRAAL [10] and TAPS [8] data. The solid line represents the result the BnGapartial wave analysis [15]. The according CBELSA/TAPS differential cross section are shown in Fig. 5.

be weaker but they may be identified due to their interference effects with the strong $S_{11}$. The $D_{13}(1520)$ shows up in the tiny curvature of the differential cross section and was finally revealed by the GRAAL beam asymmetry measurements [10]. A P-wave resonance must be present at around $1700 \mathrm{MeV}$ but its quantum numbers are uncertain. In the $\eta$-MAID model, the $P_{11}(1710)$ [16] provides a quite significant contribution in this mass range. A partial wave analysis (PWA) in the framework of the Bonn-Gatchina (BnGa) model [15], which includes data on various reactions from the CBELSA experiment as well as from other experiments, found three dominating nucleon resonances in $\eta$-photoproduction: $S_{11}(1535), P_{13}(1720)$, and in addition a newly proposed state, $D_{15}(2070)[15,9]$. In the latter analysis, the contribution of the $P_{13}(1720)$ to $N \eta$ is significantly larger than the $P_{11}(1710)$ contribution. All the existing models of $\eta$-photoproduction have in common, that they describe the existing data on differential cross sections (see Fig. 5) and on beam asymmetry (see Fig. 6) 


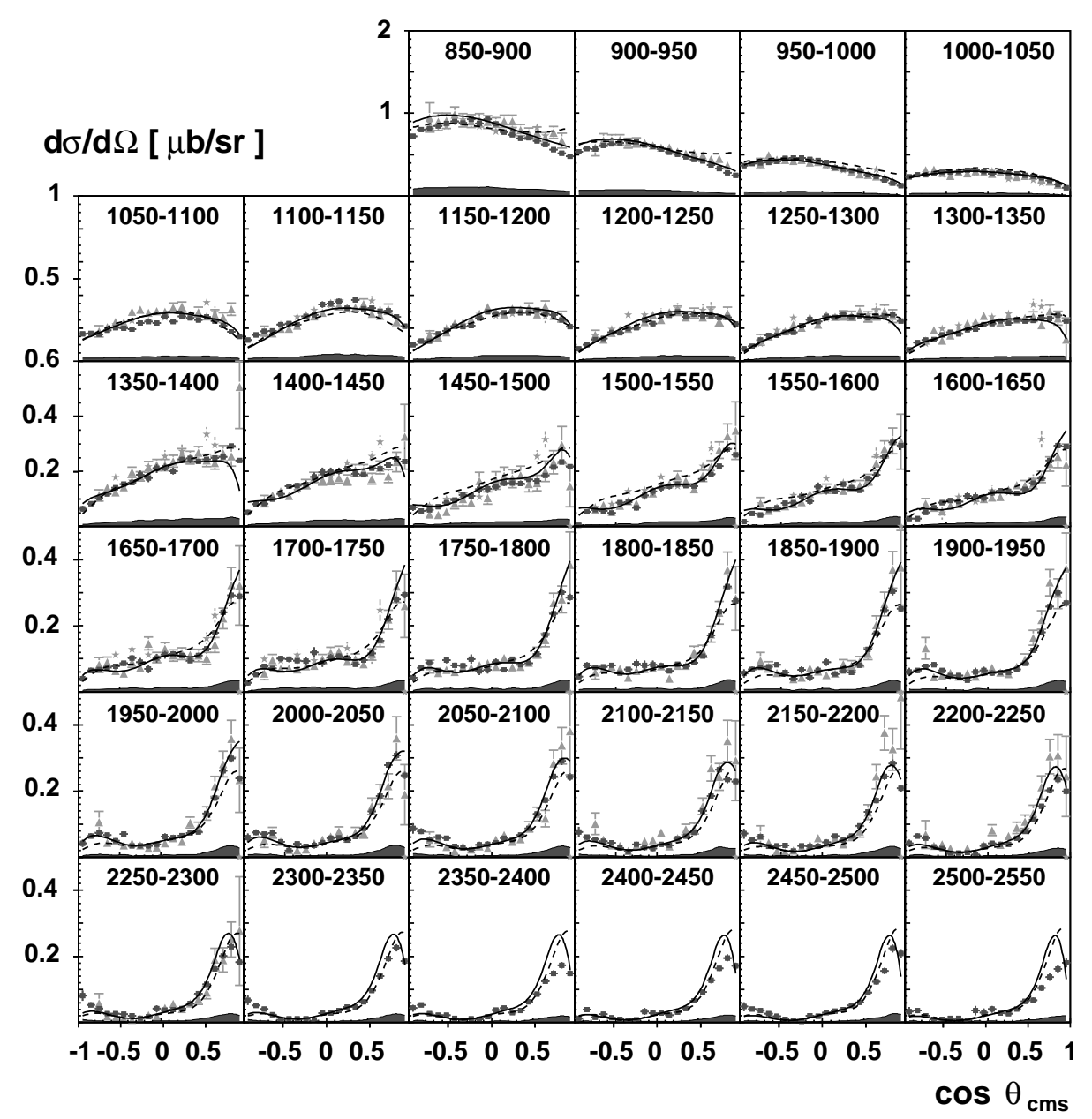

Fig. 5. Differential cross section for $\gamma p \rightarrow p \eta$ (black boxes) determined by analyzing both $\eta \rightarrow 2 \gamma$ and $\eta \rightarrow 3 \pi^{0} \rightarrow 6 \gamma$ decays (CBELSA/TAPS data [14]). In addition, data from CB-ELSA [9] (triangles) and CLAS [17] (stars) are shown. The solid line shows our previous PWA solution [15] and the dashed line represents the SAID model [18]. The data points include statistical errors only; the total systematic error is given as error bands at the bottom of each plot.
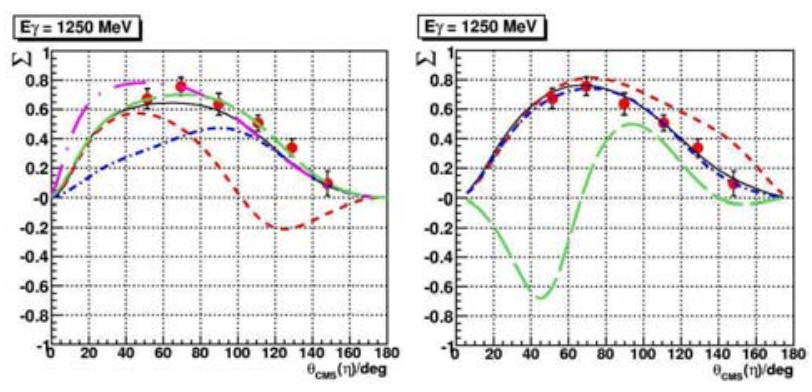

Fig. 6. Sensitivity of the $\eta$-MAID and the BnGa calculations to different resonance contributions in the beam asymmetry for the energy bin $E_{\gamma}=(1250 \pm 50) \mathrm{MeV}$. Left the $\eta$-MAID result [16] is shown, right the BnGa-PWA analysis [15]. The solid lines represent the respective full calculations. The broken curves illustrate the effect of "switching off" individual resonances; Long dashed is without $P_{13}(1720)$, long dash-dotted without $P_{11}(1710)$ (no difference to full calculation in BnGa-PWA analysis), short dashed without $D_{13}(1520)$, and short dash-dotted without $D_{15}(1675)$. rather well. Slight discrepancies between the models show up in the differential cross section at forward and backward angles and at high energies, but the error bars of the data points are too large to discriminate between the models. Also the beam asymmetry is described well in both models. As example a comparison of the CBELSA/TAPS data [19] with MAID and BnGa-PWA is shown in Fig. 6. The solid lines represent the respective full calculations. The broken curves illustrate the effect of "switching off" individual resonances; leading to very different effects in the two PWA-models. In spite of the good agreement between data and both models, the physical content of the two analyzes is rather different. In the $\mathrm{BnGa}-\mathrm{PWA}$ solution (see Fig. 6, right) the $P_{13}(1720)$ provides, in the selected mass bin, the strongest contribution. Removing $P_{13}(1720)$ in the BnGa-PWA fit (without readjustment of other parameters) a rather large effect is observed, while the effect is much smaller in $\eta$-MAID. In $\eta$-MAID fit on the other hand the $P_{11}(1710)$ significantly contributes. Switching it off leads to a significant effect in the MAID model but not 


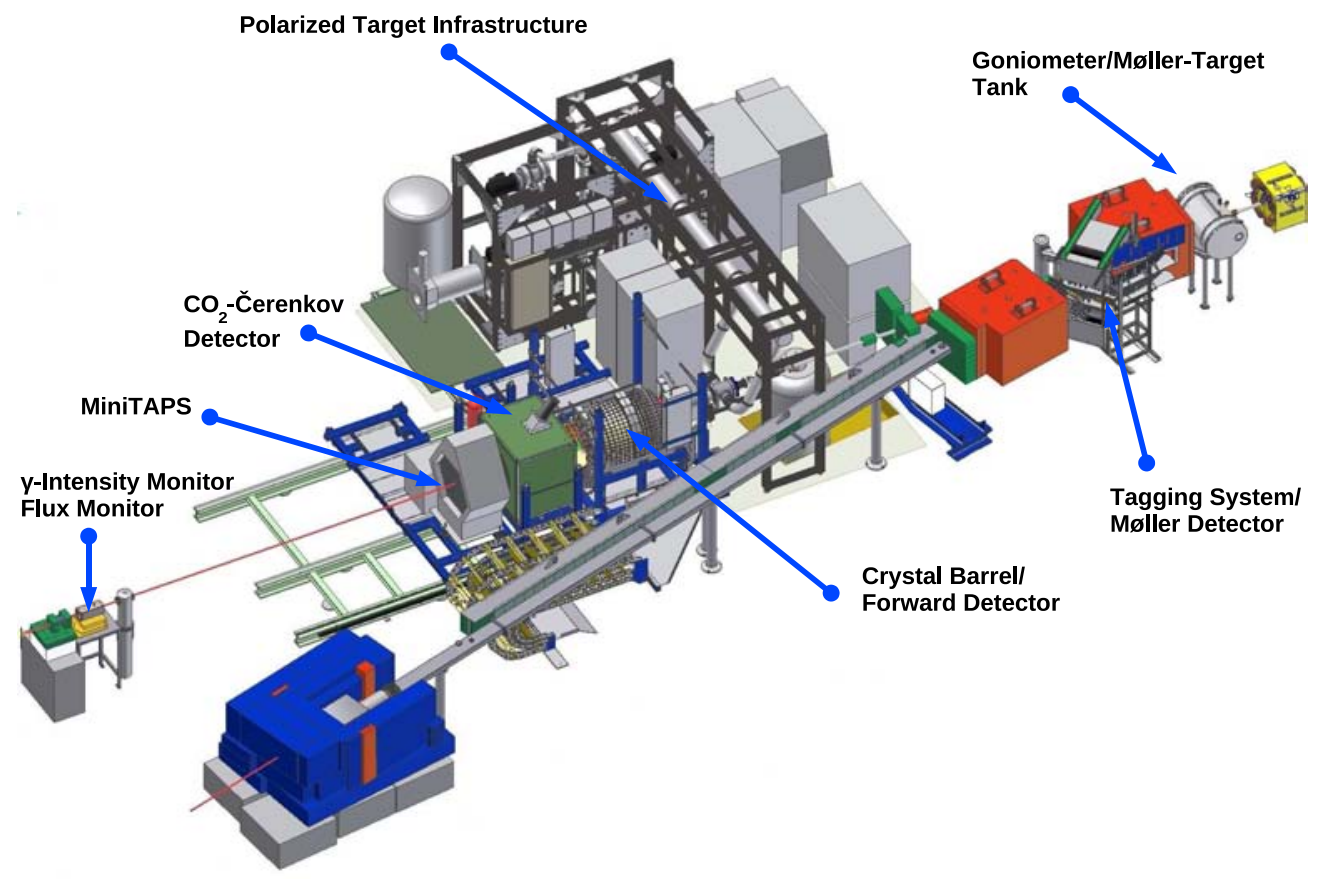

Fig. 7. Overview of the experimental setup

in the BnGa-PWA. Even though differential cross section and beam asymmetry $\Sigma$ are both well described, the contributing partial waves may still be rather different. Further observables need to be measured; observables with polarized beam and polarized target will be key measurements.

The $P_{11}(1710)$ is of course produced by one helicity amplitude only, $A_{1 / 2}$, while $A_{1 / 2}$ and $A_{3 / 2}$ lead to $P_{13}(1720)$ production. One may hence expect that the helicity differ-

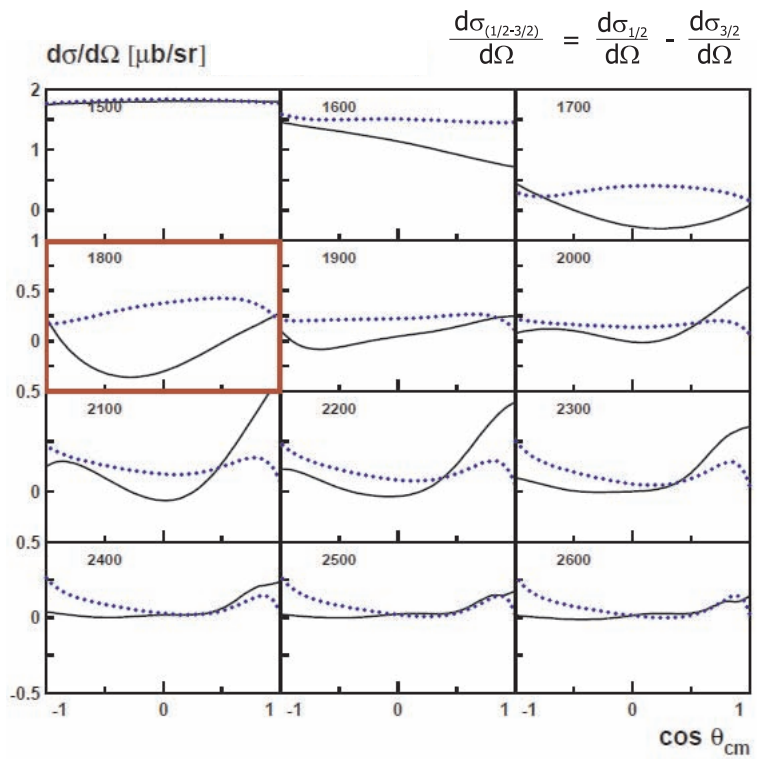

Fig. 8. Prediction of $\sigma_{1 / 2}-\sigma_{3 / 2}$ in BnGa-PWA (solid line) [15] and $\eta$-MAID (dotted line) [16]. ence $\sigma_{1 / 2}-\sigma_{3 / 2}$ is a sensitive mean to discriminate the two solutions. Figure 8 shows the prediction from $\eta$-MAID and the BnGa-PWA for $\sigma_{1 / 2}-\sigma_{3 / 2}$. In contrast to the calculations for the beam asymmetry the two solutions can be clearly distinguished here. This demonstrates the sensitivity and the strength of the double polarization observables.

\section{Experimental Setup}

The double polarization experiments have been performed with the CBELSA/TAPS experiment which is located at the accelerator facility ELSA [20] in Bonn. Figure 7 shows a sketch overview. Double polarization measurements have been performed using the Bonn-frozen-spin butanol target [21] and an energy tagged photon beam, which can be either linearly or circularly polarized. During measurements a typical mean target polarization of $70 \%$ and relaxation times around $600 \mathrm{~h}$ have been reached. Tagged electrons are provided from $18 \%$ to $96 \%$ of the primary electron energy. For the data presented here beam energies of $2.4 \mathrm{GeV}$ or $3.2 \mathrm{GeV}$ have been used. An energy resolution between $0.2 \%$ to $2.2 \%$ is achieved. Circularly polarized photons [22] have been obtained by bremsstrahlung of longitudinally polarized electrons on an amorphous radiator. At $2.4 \mathrm{GeV}$ electron energy an electron beam polarization of about $65 \%$ has been measured at the Moeller target. Linearly polarized photons are produced by using the method of coherent bremsstrahlung off a diamond radiator [23].

The Crystal Barrel calorimeter consists of $1230 \mathrm{CsI}(\mathrm{Tl})$ crystals and is complemented by two further calorimeters; the forward detector and the MiniTAPS array. The forward 


\section{$19^{\text {th }}$ International IUPAP Conference on Few-Body Problems in Physics}

detector consists of $90 \mathrm{CsI}(\mathrm{Tl})$ crystals covering the angular range between $30^{\circ}$ and $12^{\circ}$. The MiniTAPS array (216 $\mathrm{BaF}_{2}$-crystals) covers the angular range further down to $1.2^{\circ}$. While the Crystal Barrel CsI(Tl)-crystals are read out by photodiodes, the forward detector crystals are equipped with photomultipliers. This allows to obtain a fast trigger capability and time information. The time resolution achieved with this crystal-lightguide-photomultiplier configuration is about $4 \mathrm{~ns}$ (FWHM) which is sufficient for its inclusion in the first level trigger. Plastic scintillators are placed in front of the crystals of both forward detectors to identify charged particles. For the forward detector a double layer of 180 plastic scintillators of $3 \mathrm{~mm}$ thickness is placed in front of the crystals. The front faces of MiniTAPS $\mathrm{BaF}_{2}$ crystals are also covered by plastic scintillator plates. For charged particle detection a fiber detector consisting of three layers of in total 513 fibers (orientation: $\pm 25^{\circ}, 0^{\circ}$ ) is installed inside the Crystal Barrel calorimeter. This arrangement allows the reconstruction of the penetration point of the charged trajectory with the inner detector. To suppress electromagnetic background on the trigger level a $\mathrm{CO}_{2}$-Gas-Čerenkov detector has been installed between the forward detector and MiniTAPS. The Crystal Barrel/TAPS setup at ELSA is therefore very well suited to measure neutral mesons decaying into photons. The setup covers $96 \%$ of the $4 \pi$ solid angle.

\section{Recent results}

Due to the almost complete angular coverage of the CrystalBarrel/TAPS experiment, cross sections and polarization variables can be measured over the full solid angle. Apart from the differential cross section, the polarization observables $\Sigma$ and $G$ have been measured using linearly polarized photons and a longitudinally polarized target, while $E$ was determined using circularly polarized photons impinging on a longitudinally polarized target.

\subsection{Measurements with circularly polarized photons}

Fig. 9 and 10 give an impression of the quality of the double polarization data taken with the CBELSA/TAPS experiment at ELSA. Even though data selection and calibration is still preliminary, Fig. 9 shows clear $\pi^{0}$ - and $\eta$-signals above a small background, which will be reduced further by a more refined analysis. The missing mass distribution for events $\vec{\gamma} \vec{p} \rightarrow X \eta$, using an $\eta$ cut as indicated in Fig. 9 gives a clearly visible peak at the mass of a proton. The two curves shown in Fig. 10 give a comparison of the missing mass spectrum for events with a polarized beam where the spin of photon is parallel or anti-parallel to the spin of the proton. For events with a missing mass being consistent with a proton, a clear count rate difference $N_{1 / 2}-N_{3 / 2}$ is observed; the events with helicity $1 / 2$ dominate over the events with helicity $3 / 2$. This is more clearly seen if the difference is plotted, which is shown in Fig. 11. Nicely visible is a peak at the mass of the proton. The other figure in 11 shows the difference of the missing mass spectra for the

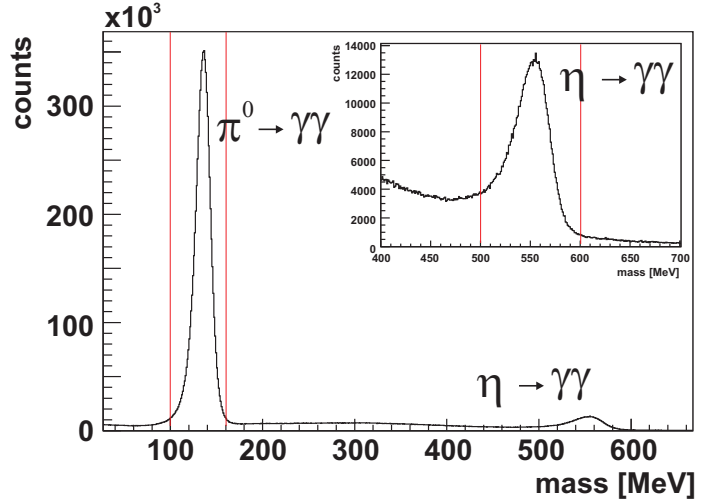

Fig. 9. Invariant $\gamma \gamma$-mass distribution for events with a charged particle and two photons in the final state after a coplanarity and a missing mass cut was performed. Analysis and calibration: preliminary (only part of the statistics shown). The vertical (red) lines indicate the $\pi^{0}$ - and $\eta$-mass cut used.

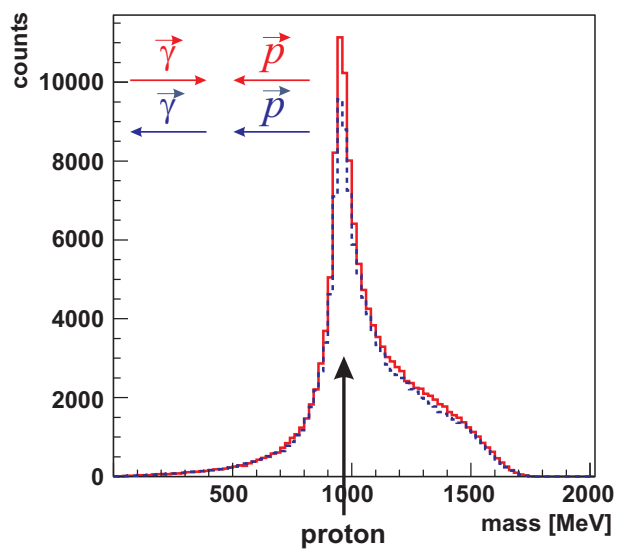

Fig. 10. Missing mass distribution for events which are consistent with $\vec{\gamma} \vec{p} \rightarrow X \eta$ after having performed a cut on the mass of the $\eta$ and a coplanarity cut. Events with helicity $1 / 2$ are plotted as solid (red) line, events with helicity $3 / 2$ as dashed (blue) line. A clear count rate difference is observed in the proton mass region. Preliminary analysis and calibration, only part of the statistics shown

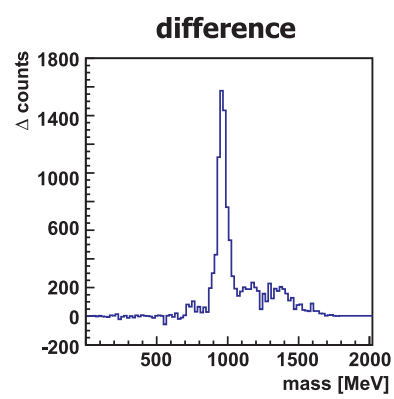

unpolarised difference

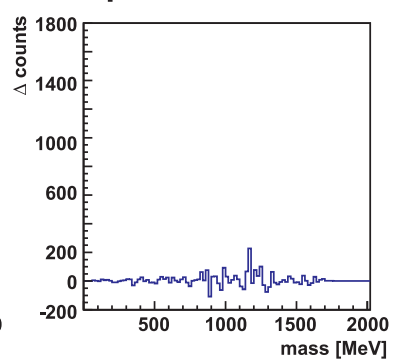

Fig. 11. Left: Difference of the missing mass spectra with parallel and anti-parallel orientation of the proton spin and the photon spin. Right: Cross check of the data, plotting the unpolarized difference by adding up spills with opposite spin.

unpolarized case as a crosscheck. This is done by summing up the events for both spin states. 


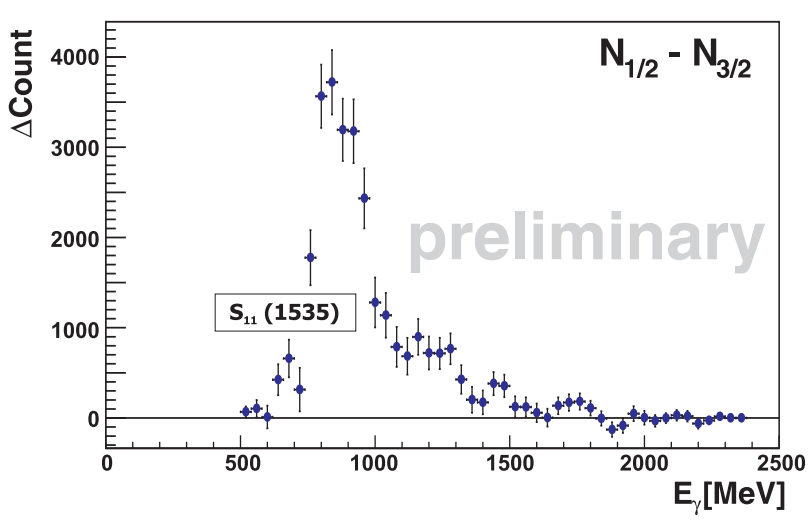

Fig. 12. Raw count rate differences between events with helicity $1 / 2$ and $3 / 2\left(\Delta=N_{1 / 2}-N_{3 / 2}\right)$ for the reaction $\vec{\gamma} \vec{p} \rightarrow p \eta$. This data does so far neither contain corrections for acceptance nor has the polarization and photon flux been taken into account hence its status is very preliminary.

As expected, the anti-parallel orientation of the proton and photon spin $\left(N_{1 / 2}\right)$ dominates at low energies (see Fig. 12). This is due to the dominance of the $S_{11}(1535)$ close to threshold. The behavior is also nicely visible in the PWA-predictions for $\sigma_{1 / 2}-\sigma_{3 / 2}$ shown in Fig. 14. Clear deviations between the different PWA-solutions are observed above photon energies of $E_{\gamma}=1000 \mathrm{MeV}$. The region around $E_{\gamma}=1100 \mathrm{MeV}$ is of particular interest. Here, the different PWA-solutions make clearly distinct predictions. While the solution of the BnGa-PWA published in $[15,9,5]$ allows, within errors, for negative values in $\sigma_{1 / 2}-$ $\sigma_{3 / 2}$ up to slightly positive values, the $\eta$-MAID solution shows a clear positive value (Fig. 14). The previously mentioned difference between the BnGa-PWA and $\eta$-MAID solution is a strong contribution of the $P_{11}(1710)$ in $\eta$ MAID, which contributes less in the BnGa-PWA, where $P_{13}(1720)$ contributes quite strongly. A very preliminary comparison with the data, which are not corrected for acceptance, nor has the polarization or the flux been taken into account, indicates that the helicity difference might indeed be positive. More information will be provided by the according differential distributions. Significant differences in the PWA-predictions are also observed at higher energies. Also here the new data will make a significant impact.

Fig. 13 shows the corresponding distributions for the $p \pi^{0}$ final state. The energy dependent count rate difference $\mathrm{N}_{1 / 2}-\mathrm{N}_{3 / 2}$ indicates resonance structures, starting at low energies, where the $\Delta(1232)$ dominates. As expected, negative values are found in the $\Delta(1232)$ region. In the second and third resonance region the present raw count rate difference (preliminary, uncorrected for acceptance, finite polarization, and photon flux) indicates positive and negative values, respectively. Figure 15 shows significant differences between the different PWA-models. The sensitivity of $\sigma_{1 / 2}-\sigma_{3 / 2}$ on the differences in the PWA, already visible in the total cross section, is much more pronounced in the differential distributions. Again an almost complete angular coverage will be reached for the differential dis-

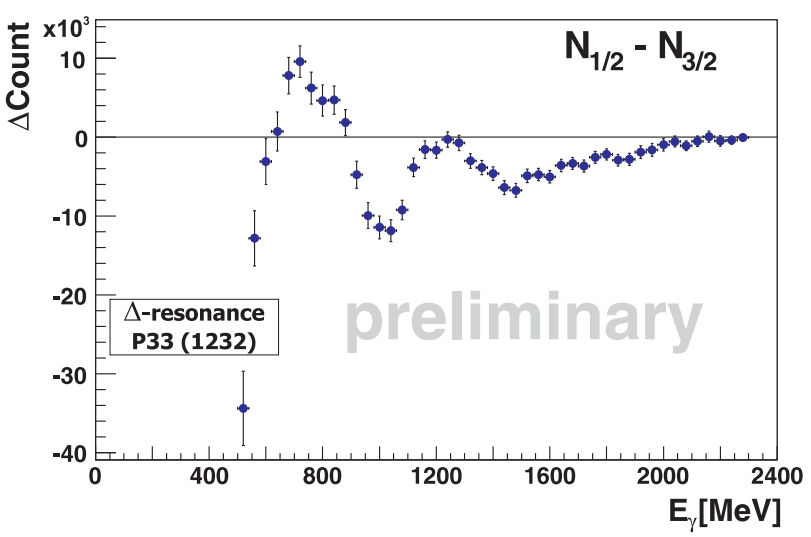

Fig. 13. Raw count rate differences between events with helicity $1 / 2$ and $3 / 2\left(\Delta=N_{1 / 2}-N_{3 / 2}\right)$ for the reaction $\vec{\gamma} \vec{p} \rightarrow p \pi^{0}$. This data does neither contain corrections for acceptance nor has the polarization and photon flux been taken into account hence its status is very preliminary [24].

tribution. This information is of course very valuable to further constrain the PWA-models.

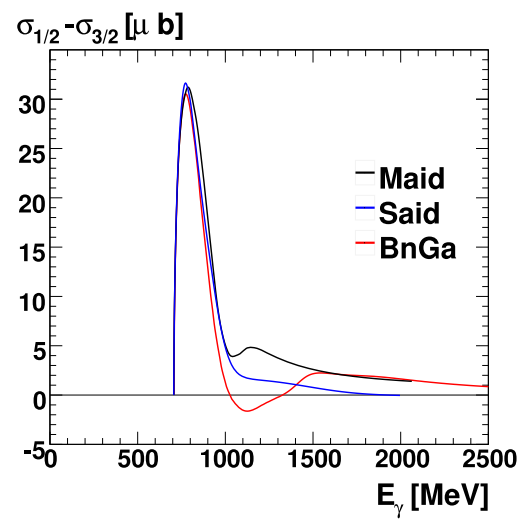

Fig. 14. Predictions for the helicity difference $\sigma_{1 / 2}-\sigma_{3 / 2}$ from the BnGa-PWA, MAID and SAID models.

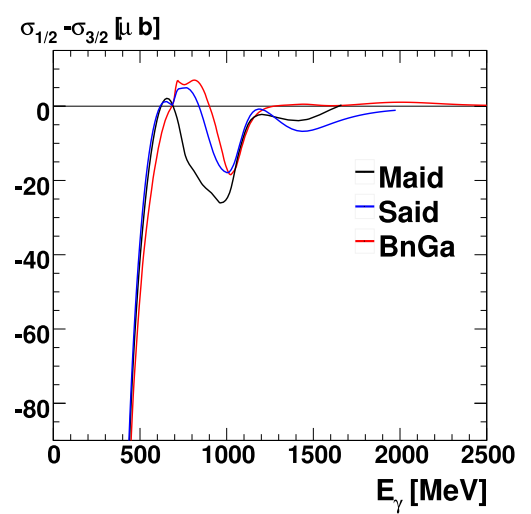

Fig. 15. Predictions for the helicity difference $\sigma_{1 / 2}-\sigma_{3 / 2}$ from the BnGa-PWA, MAID and SAID models 


\section{$19^{\text {th }}$ International IUPAP Conference on Few-Body Problems in Physics}

\subsection{Measurements with linearly polarized photons}

Using linearly polarized photons and a longitudinally polarized target the single polarization observable $\Sigma$ and the double polarization observable $G$ can be measured simultaneously. Data were taken recently at ELSA, that cover a range in the photon energy from $E_{\gamma}=0.4$ to $1.35 \mathrm{GeV}$. Fig. 16 shows the intensity and polarization degree for two

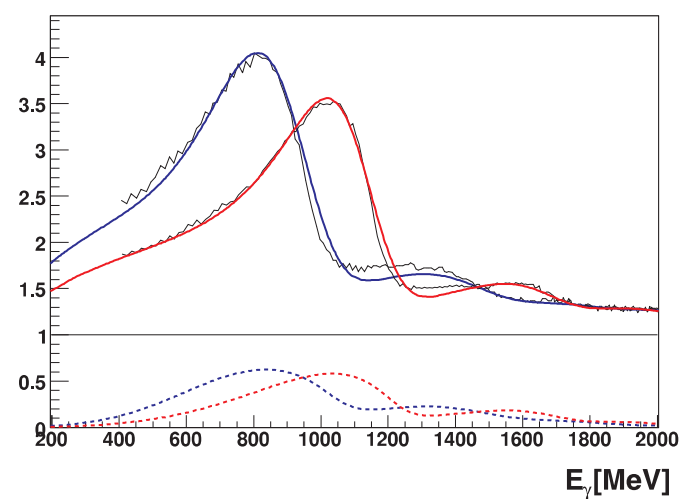

Fig. 16. Intensity spectra (full lines) and polarization degrees (dashed lines) for two different diamond settings. The maxima of the coherent peaks are at 840 and $1032 \mathrm{MeV}$.

diamond radiator orientations as a function of the photon energy. In the preliminary analysis the $\eta$ and the $\pi^{0}$ are reconstructed from events with 3 clusters in the calorimeter. In experiments with linear polarized photons, the polarization vector fixes a second axis in addition to the beam direction. The reaction is no longer $\phi$-symmetric and a modulation in the azimuthal angle $\phi$ can be observed, as one

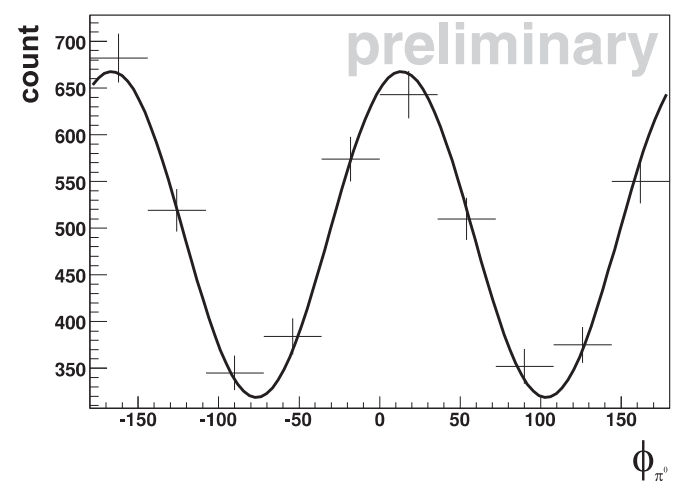

Fig. 17. $\phi$ distribution of the $\pi^{0}$ in the reaction $\vec{\gamma} \vec{p} \rightarrow p \pi^{0}$. For the definition of the $\phi$-angle see Fig. 3. (y-axis zero suppressed)

can see in Fig. 17. The measured distribution can be described by the following equation:

$$
\begin{aligned}
N(\theta, \phi)=\left(N_{C}+N_{H}\right) & \cdot\left(1-\Sigma \cdot p_{\text {lin }}^{\gamma} \cos (2 \phi)\right) \\
+ & \frac{N_{H}}{N_{C}+N_{H}} \cdot p_{z}^{\text {target }} p_{\text {lin }}^{\gamma} \cdot G \cdot \sin (2 \phi)
\end{aligned}
$$

The polarization observable $G$ can be extracted by fitting the observed $\phi$-dependence within a certain $\theta$-bin by the function

$$
N(\theta, \phi)=A \cdot(1-B \cdot \cos (2 \phi)+C \cdot \sin (2 \phi))
$$

The parameter $\mathrm{C}$ of the fit allows the extraction of the double polarization observable $G$, if also the target polarization, the degree of linear polarization of the beam and the dilution factor $\frac{N_{H}}{N_{C}+N_{H}}$ is known. A preliminary dilution factor has been determined from an additional analysis of data which has been taken using a hydrogen and a carbon target. Figures 18 and 19 show examples of the obtained preliminary results [25] taking into account a preliminary dilution factor. Again the results are shown in comparison to the partial wave predictions from the BnGa-PWA, MAID and SAID and again significant differences in the predictions of the different models are observed. Clearly the new data will provide important new information for the PWA. Due to the fact, that presently only events are analyzed where also a cluster due to the proton was observed in the calorimeters, the angular coverage is not complete. This will be significantly improved by a more refined analysis.

These first measurements of the double polarization observables $G$ will as well as the double polarization observable $E$ provide significant new constraints for the PWA.

\section{Outlook}

The new single and double polarization data obtained with the Crystal-Barrel/TAPS experiment at ELSA will provide new important information for the partial wave analysis. They will resolve ambiguities in the present solutions and reduce model-dependent uncertainties in the extraction of nucleon resonance parameters, providing a new benchmark for comparisons with QCD-inspired models and lattice calculations.

We acknowledge financial support from the Deutsche Forschungsgemeinschaft (SFB/TR16).

\section{References}

1. C. Amsler et al. (Particle Data Group), Physics Letters B667, 1 (2008)

2. C. Wu et al. (The SAPHIR-Collaboration), Eur. Phys. J. A 23 (2) (2005)

3. A. Braghieri et al., Phys. Lett. B551, 46 (1995).

4. M. Wolf et al. [TAPS collaboration], Eur. Phys. J. A 9, 5 (2000).

5. U. Thoma, M. Fuchs et al. [CB-ELSA collaboration], Phys. Lett. B 659 (2008) 87

6. M. Fuchs, PhD. thesis Photoproduktion neutraler Pionpaare mit dem Crystal Barrel Detektor an ELSA. (2005) 

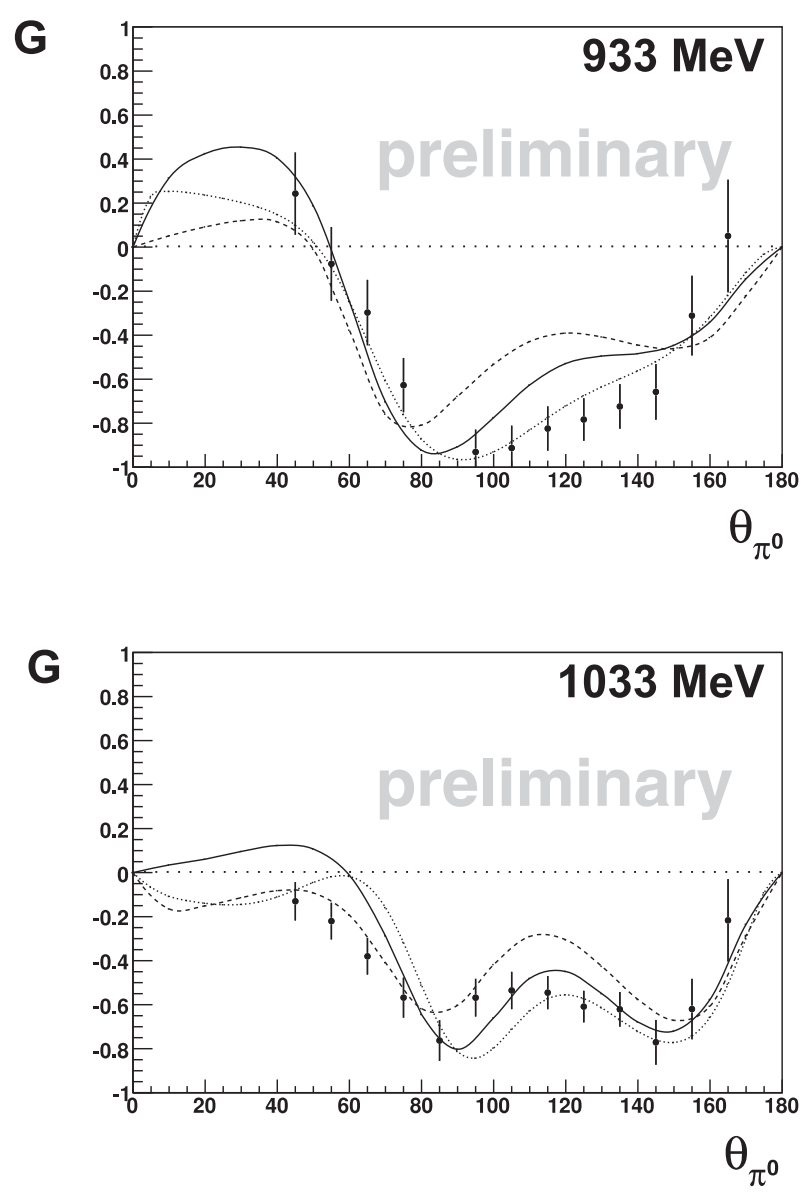

Fig. 18. Preliminary results for the double polarization observable $G$ for the reaction $\vec{\gamma} \vec{p} \rightarrow p \pi^{0}$ with a preliminary dilution factor taken into account. The two energy bins show have a width of $33 \mathrm{MeV}$. Solid line: SAID, dashed line: MAID, dotted line: BnGa-PWA

7. H. van Pee et al., Eur. Phys. J. A 31 (2007) 61

8. B. Krusche et al. [TAPS collaboration], Phys. Rev. Lett. 74, 3736 (1995).

9. V. Crede et al. [CB-ELSA Collaboration], Phys. Rev. Lett. 94, 012004 (2005)

10. J. Ajaka et al. [GRAAL collaboration], Phys. Rev. Lett. 81, 1797 (1998).

11. I. Horn et al. [CB-ELSA Collaboration], Phys. Rev. Lett. 101 (2008) 202002

12. I. Horn et al. [CB-ELSA Collaboration], Eur. Phys. J. A 38 (2008) 173

13. W. T. Chiang and F. Tabakin, Phys. Rev. C 55, 2054 (1997)

14. V. Crede et al. [CBELSA/TAPS Collaboration], Phys. Rev. C 80, 055202 (2009)

15. A. V. Anisovich, A. Sarantsev, O. Bartholomy, E. Klempt, V. A. Nikonov and U. Thoma, Eur. Phys. J. A 25, 427 (2005)

16. W. T. Chiang, S. N. Yang, L. Tiator, M. Vanderhaeghen and D. Drechsel, Phys. Rev. C 68, 045202 (2003)

17. M. Dugger et al. [CLAS Collaboration], Phys. Rev. Lett. 89, 222002 (2002) [Erratum-ibid. 89, 249904
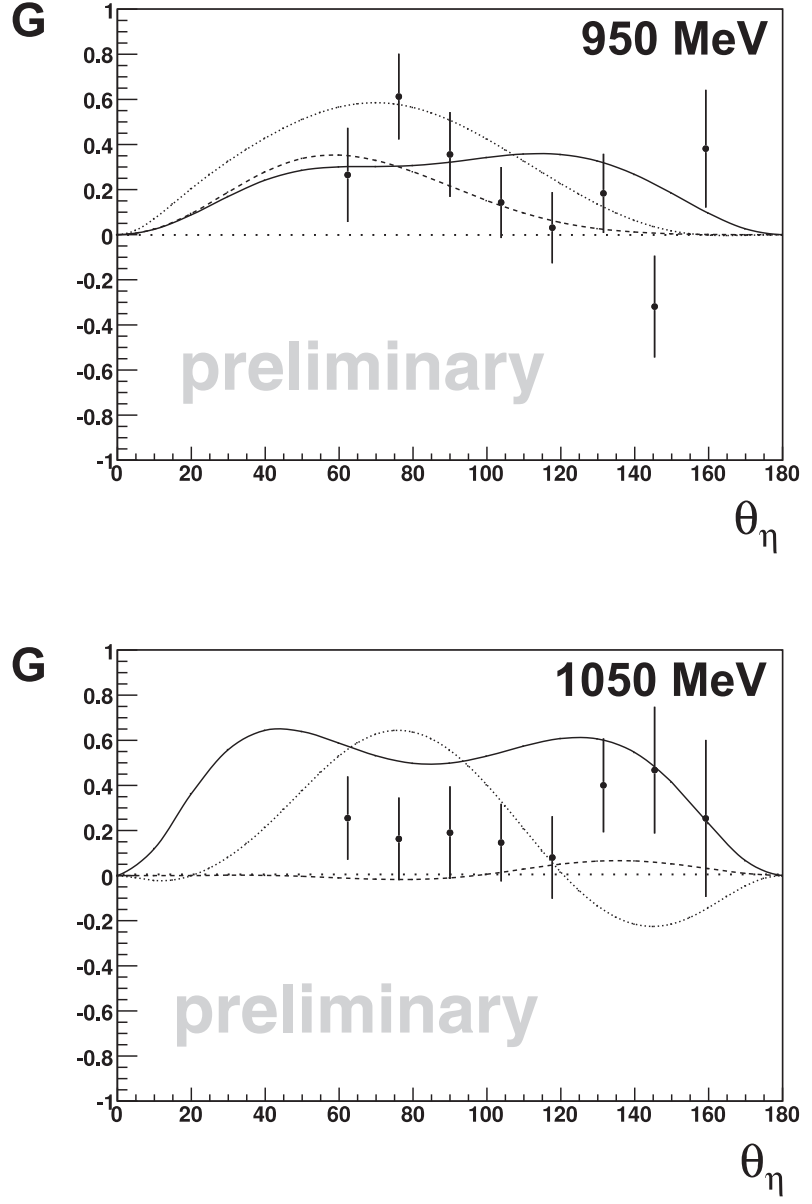

Fig. 19. Preliminary results for the double polarization observable $G$ for the reaction $\vec{\gamma} \vec{p} \rightarrow p \eta$ with a preliminary dilution factor taken into account. The two energy bins show have a width of $33 \mathrm{MeV}$. Solid line: SAID, dashed line: MAID, dotted line: BnGa-PWA

(2002)].

18. R. A. Arndt, W. J. Briscoe, I. I. Strakovsky and R. L. Workman, Phys. Rev. C 66, 055213 (2002)

19. D. Elsner et al., Eur. Phys. J. A 33 (2007) 147

20. W. Hillert, Eur. Phys. J. A 28 (2006) 139

21. Bradtke et al, NIM A 436(1999) 430

22. Susanne Kammer, PhD. thesis Strahlpolarimetrie am CBELSA/TAPS Experiment (2010)

23. D. Elsner et al, Eur. Phys. J. A39 373-381 (2009)

24. M. Gottschall, $\mathrm{PhD}$. thesis (in preparation), Bonn (2009)

25. A. Thiel, $\mathrm{PhD}$. thesis (in preparation), Bonn (2009) 